[CONTRibution From the Food Investigation Laboratory, BuREaU of Chemistry, UNited STATES DEPARTMENT OF AgRiculture.]

\title{
A METHOD FOR THE EXAMINATION OF METHYL SALICYLATE.
}

By Alan R. Albright.

Received March 2, 1917.

In former years, artificial salicylic acid, and in consequence its synthetic esters, were to be found on the market in very impure condition. There were present usually considerable quantities of phenol, $p$-hydroxybenzoic acid, and cresotic acids, or their respective esters. As methods of manufacture have been improved, the degree of freedom from such undesirable accompanying substances has been raised, until at present these materials are available in probably as pure a state as any synthetic compounds of like physical character; that perfection has not even yet been attained, however, will be brought out later in this paper.

More recently, an extended use has been made of synthetic methyl salicylate, both for medicinal and for flavoring purposes, gradually increasing until the natural oils of wintergreen and of sweet birch, consisting almost exclusively of methyl salicylate, ${ }^{1}$ have become relatively unknown. The cause of this has been two-fold: first, the high quality of the synthetic ester, and second, the strict enforcement of the National food and drugs act. Many firms, therefore, have ceased altogether the handling of any oil labeled genuine, which is evidence that before the passage of that statute in 1906 much of the supposed natural oil was in reality artificial. Demand for the natural product has persisted in some quarters, however, and this, coupled with its scarcity, has put a premium upon it, which in turn has increased the temptation to practice fraud, since the relative prices of synthetic methyl salicylate and oil of wintergreen, shortly before the European war, were about I : I4 (\$o.30:\$4.25 per 1b.). ${ }^{2}$ The names of the natural oils have been retained, but the materials sold under them may range in composition from the rare genuine oil to an entirely synthetic product. In the latter case, the designation is usually appropriately qualified; in the case of mixtures, it is frequently not so, and it is on account of this circumstance that the Federal food and drugs act has on several occasions been invoked to prevent deception.

The purpose of this investigation of some of the synthetic methyl salicylates found upon the market has been to devise a means for the detection of the possible impurities that in very minute amounts may still

${ }^{1}$ Power and Kleber, Pharm. Rundschau, 13, 228 (1895).

2 In November, I915, manufactured methyl salicylate had reached the remarkably high price of \$4.Io per $1 \mathrm{~b}$., that of oil of wintergreen remaining approximately stationary. At the present time, February $26,19 \times 7$, the market price of synthetic methyl salicylate is $\$ 0.80-\$ 0.90$ per lb.; oil of birch is quoted at $\$ 2.50-\$ 2.75$, and oil of wintergreen at $\$ 3.85-\$ 4.00$ per lb. 
be looked for in them, and to discover by parallel experiments whether these same compounds exist in the natural oils of wintergreen and birch.

Means have been devised in the past for the recognition of pure oils of birch and of wintergreen. Examples of these are the color tests given when the oils are treated with vanillin and sulfuric acid ${ }^{1}$ or by very similar reactions, using, respectively, piperonal and chloral hydrate solution in place of vanillin. ${ }^{2}$

The odors of the genuine oils are characteristic, but only a practiced olfactory sense can detect adulteration in this way.

Early attempts to detect impurities in the synthetic products were easily successful, but this object has more lately become exceedingly difficult of attainment. For example, Kolbe, in one of his earliest papers, ${ }^{3}$ mentions the fact that on examining under the microscope some of his salicylic acid he was able instantly to recognize crystals of $p$-hydroxybenzoic acid, which crystallizes, as he says, in small rhombic tables. This fact has been published again by Henry L. Smith, ${ }^{4}$ who, in order to make the para-acid more readily distinguishable, concentrates it, making use of the difference between the solubility of its basic calcium salt and that of the corresponding salicylic acid compound. Smith states that it is scarcely possible to detect microscopically $\mathrm{r} \%$ of the para-acid in salicylic acid, without this partial isolation.

Englehardt and Jones ${ }^{5}$ have applied Carletti's ${ }^{6}$ test for phenols in salicylic acid and salicylates, which depends on the fact that a characteristic color is produced on treating a water solution of the acid or one of its salts with a small quantity of a dilute solution of furfural and adding concentrated $\mathrm{H}_{2} \mathrm{SO}_{4}$. Englehardt and Jones were able in this way to detect an addition of $0.15 \%$ of $o$-cresol; out of fifteen samples of synthetic methyl salicylate, they state that four showed the presence of a phenol; and of four samples of natural oil of wintergreen, three gave positive tests for phenol.

In Allen's "Commercial Organic Analysis," Vol. III (4th edition), are given accounts of further tests, which, however, deal with cases involving the presence of larger proportions of the respective impurities.

The method which is here presented is based upon differences in some optical properties of crystalline derivatives of methyl salicylate and of the most probable impurities occurring in it. The-derivative selected was

${ }^{1}$ Described, with reference to a test for acetone, by Kutscheroff, $Z$. anal. Chem., 44, 622 (1905); amplified in the Bur. of Chem. Iaboratories.

${ }^{2}$ Watson and Sayre, J. Amer. Pharm. Assoc., 3, 1658 (1914).

2 J. für prakt. Chem., [2] 10, 89-1 I2 (especially p. 98) (1874).

- Analyst, 41, 3-6 (1916).

B Proc. Am. Pharm. Assoc., 56, 866 (1908).

B Boll. chim. farm., 46, 42 I (1907); Schweiz. Apoth. Ztg., 45, 702 (1907); Deut. A poth. Ztg., No. I2 (I g08). 
the benzoate, since benzoyl methyl salicylate, phenyl benzoate, $m$ - and $p$-cresyl benzoates, benzoyl p-hydroxybenzoic acid methyl ester, and benzoyl $p$-cresotic acid methyl ester are all easily prepared under like conditions and in general crystallize readily from the same solvents.

These substances were first prepared in the pure state, by applying the Schotten-Baumann reaction, after esterification in the cases of the methyl esters. Usually 3-5 g. of the oil or solid were placed in a $250 \mathrm{cc}$. flask, an excess of benzoyl chloride added, and the mixture kept in thorough agitation and made alkaline from time to time with dilute $\mathrm{KOH}$, until the reaction was complete. The resulting crystalline precipitate was filtered off on a Büchner funnel, the lumps crushed, washed several times with water, and dried. Each substance was then recrystallized from a large bulk of slightly warm ethyl alcohol diluted to such an extent that the solution became supersaturated at only a little above room temperature. Under these conditions minute crystals separated not too rapidly and very nearly all in perfect form. Each compound in turn was then studied under the microscope, giving attention especially to the determinations of habit, refractive index and extinction angle, in order to provide criteria by which later to identify these susbstances when found in admixture with other compounds.

It may be of interest and not superfluous here to state, regarding the refractive indices of these materials, that of a mixture of two substances having different indices of refraction, the one, if isometric, may be rendered practically invisible by immersing the mixture in a liquid possessing the same refringence as that substance. When a transparent or translucent object is immersed in a liquid which has a refractive index either higher or lower than that of the solid and is examined under the microscope, using correct illumination, there are observed so-called contour bands consisting of dark lines bounding the particle, paralleled by bright bands, or "halos." After focussing sharply on the edge of the crystal, if the objective be very slightly and gradually raised, these bright bands will appear to move in the direction of the substance of higher refractive index; $i . e$. , they will move in, converge, toward the center of the crystal if that have the higher index, or out into the mounting medium if that be higher in refractive power. When both crystal and liquid have the same refractive index, the contour bands will practically disappear, the edges of the crystals appear to merge into the liquid, and the particle become almost if not entirely invisible. Then any substance of either higher or lower index will stand out in more or less sharp relief. We have then what may be termed a method of optical concentration of the second substance. The fact must be considered, however, that benzoyl methyl salicylate, as well as each of the other compounds under discussion, shows birefringence, $i$. e., possesses two principal refractive indices depending 
upon the direction in which the axes of the crystal lie with reference to the path of the ray of polarized light used for illumination. It is satisfactory for the present purpose of examination, however, to use for mounting a solution of refractive index equal to the mean of the two principal indices $(\alpha$ and $\gamma$ ) of benzoyl methyl salicylate, viz.: 1.658 , the mean of I. 698 and I. 6 I8. The liquid used in this case was a solution of mercuric potassium iodide in water, Sonstadt's, or Thoulet's, solution, which, by careful dilution or concentration is capable of adjustment to any refractive index from practically that of water up to above $I .7 I$ at room temperature. When, then, crystals of the orthorhombic benzoyl methyl salicylate, having a relatively high mean refractive index, admixed with a small proportion of monoclinic phenyl benzoate of lower index, are mounted in Thoulet's solution of refractive index, 1.658, the diamondshaped salicylate derivative tends to disappear, while the needles of the latter substance become more readily distinguishable. If any needles at all stand out in clear view under these circumstances, they probably consist of phenyl benzoate, but the possibility is to be kept in mind that they may be fragments of the methyl salicylate derivative, still visible on account of some flaw in structure. The habit of phenyl benzoate is generally sufficiently characteristic for its identification by simple observation, but, in order to avoid confusing the one with the other, the angle of extinction of the crystal under examination is measured, since the numerical values of this property are decidedly different for the two compounds. This measurement is carried out in the following way: A polarizing petrographical microscope is used, with the usual cross-hair ocular, fixed in position, one of the cross-hairs being parallel to the plane of light. The nicols are crossed, and the preparation centered on the revolving stage, with one of the needles of phenyl benzoate near the center of the field, and the stage rotated until the crystal rests with its long axis parallel to one of the cross-hairs. Under these conditions the crystal appears brightly illuminated, but on rotating the stage again, the particle "extinguishes" at an angle oblique to either of the cross-hairs $\left(30^{-} 33^{\circ}\right)$. This oblique extinction is a property of monoclinic crystals, and by its aid they may readily be distinguished from the orthorhombic benzoyl methyl salicylate, which exhibits parallel extinction, $i$. e., the latter crystals are entirely invisible between crossed nicols when their long axes are parallel to either cross-hair, but most brilliantly illuminated at a point half-way between them. ${ }^{1}$

On applying this method in the examination of mixtures of the purest methyl salicylate obtainable with different amounts of phenol the crys-

${ }^{1}$ See Wright, Chamot, Weinschenk-Clark, or Johannsen for very satisfactory accounts of these optical phenomena and of the use of oblique illumination, and other interesting aids. See aiso F. F. Wright on "The Petrographic Microscope in Analysis," This Journal, 38, 1647-58 (1916). 
tals of phenyl benzoate could be readily distinguished as they showed the same habit, relative refractive index and extinction angle as when observed without the admixture of the methyl salicylate derivative. Microscopical quantitative measurements of the percentage of phenol present gave unsatisfactory results.

On benzoylating and recrystallizing the benzoate of several commercial samples of synthetic methyl salicylate, optical evidence of the presence of very minute amounts of phenol was obtained in every case; furthermore, this substance was detected in the same way in each of a number of unquestionably genuine samples of oils of wintergreen and sweet birch, the quantity of phenol being apparently very slightly greater in the natural than in the synthetic products.

The assertion is not made that this is a rigid proof of the presence of phenol. However, if present at all, the amounts occurring are so very minute that only some such test as an optical or exceedingly delicate color reaction seems capable of being applied. The microscopical evidence is believed, however, to be as conclusive as any which can be brought forward; and accepting it, phenol in traces may be considered to be a normal constituent of both the synthetic and natural oils.

Regarding the origin of this compound in the several types of methyl. salicylate oils; it is possible that phenol is formed in small quantity during the distillation or rectification of the oils, as the result of a partial saponification of the ester by steam, followed by a breaking-down of the salicylic acid by the action of heat, into carbon dioxide, and phenol. ${ }^{1}$ Since, furthermore, the production of these oils invariably involves their subjection to the action of water vapor at approximately $100^{\circ}$, the author feels justified in using the term normal.

Preparations of benzoylated artificial and natural methyl salicylate oils were then closely examined with the object of finding impurities or accompanying substances other than phenol, if present.

In derivatives of some synthetic oils, clear evidence was obtained of the presence of very minute quantities of the benzoate of $p$-cresotic acid methyl ester $\left(\mathrm{C}_{6} \mathrm{H}_{3}\left(\mathrm{O}-\mathrm{OC}-\mathrm{C}_{6} \mathrm{H}_{5}\right)\left(\mathrm{CO}-\mathrm{OCH}_{3}\right)\left(\mathrm{CH}_{8}\right)_{1,2,4}\right)$. Systematic and thorough search failed to show this compound occurring in any of the benzoates of the genuine oils. It is therefore to be assumed, when this substance is found in a sample of unknown origin, that that sample is at least in part artificial, and that the synthetic methyl salicylate with which it was mixed, was manufactured without proper care.

As to the methyl ester of benzoyl p-hydroxy benzoic acid, this compound is difficult to obtain in perfect crystalline form by the method described; it continually separates in very thin scales having scarcely any angle definition, so that its recognition is accompanied by doubt. Crystals

${ }^{1}$ H. D. Gibbs, Phil. Jour. of Science, Sec. A, 3, IOI-9 (especially p. 102) (1908). 
exactly resembling them, however, were found occasionally in some synthetic methyl salicylate derivatives; on the other hand, they seemed lacking in all of the products from the natural oils. The evidence here is too inconclusive, however, to warrant making the definite statement that synthetic methyl salicylate is accompanied by its para-isomer.

Considering the high degree of purity that has been noticed during the examination of artificial methyl salicylates from various sources of manufacture, the conviction has been reached that, in the course of time, the examination of methyl salicylate oils will have for its object not so much the distinguishing of natural from synthetic oil, as the detection of those impurities in methyl salicylate to which have been ascribed certain undesirable physiological effects. It appears to be possible at the present time to manufacture methyl salicylate entirely free from such obnoxious by-products. When all interested manufacturers produce this material with the same care, there should be no reason for further adulteration.

To summarize:

As a means of examining a methyl salicylate oil, the main constituent of such oil and all other phenolic accompanying substances or impurities are converted into the same crystalline derivative-the benzoate. This product, after recrystallization, is then mounted on an object slide, using a liquid equal in refractive index to the mean index of benzoyl methyl salicylate. Under these conditions, when examined with a petrographic microscope, the salicylate derivative tends to become invisible, leaving other compounds in clearer view, so that their optical properties may be more readily observed and their identification thus facilitated.

Using this method, evidence has been found of the presence of phenol in both genuine and imitation oils of wintergreen and sweet birch. In some synthetic methyl salicylates, indications have been had of the presence of an exceedingly small amount of its next homolog, viz., p-cresotic acid methyl ester, whereas no trace of this compound has been found in a natural oil. A substance closely resembling an isomer of methyl salicylate, methyl p-hydroxybenzoate, occurs in the synthetic ester, but appears not to exist in the genuine oils of wintergreen or birch.

It is believed that, in time, further improvements in purification pro cesses will lead to the production of synthetic methyl salicylate of such a high quality that the use of the natural-and expensive-oils of wintergreen and birch will be entirely superseded by that of the artificial.

The writer wishes to express his thanks for very courteous aid, to Dr. H. E. Merwin, of the Geophysical Laboratory, and Mr. E. S. Larsen, of the Geological Survey; and to acknowledge especially his indebtedness to Dr. E. T. Wherry, of the National Museum, for his very kind and painstaking assistance.

WASEINGTON. D. C 